Karstenia 53: 9-28, 2013

\title{
Collections of Hygrocybe subsect. Squamulosae from N. Finland, N. Norway, Arctic Canada and Arctic Russia (Polar Urals)
}

\author{
TORBJØRN BORGEN and ESTERI OHENOJA
}

\begin{abstract}
BORGEN, T. \& OHENOJA, E. 2013: Collections of Hygrocybe subsect. Squamulosae from N. Finland, N. Norway, Arctic Canada and Arctic Russia (Polar Urals). - Karstenia 53: 00-00. HELSINKI. ISSN 0453-3402.
\end{abstract}

\begin{abstract}
About 80 collections of Hygrocybe, subsect. Squamulosae, collected in the Arctic and montane areas in N. Europe, Canada and Siberia were studied macro- and microscopically, based on personal collections and herbarium material. Seven species are recognized, viz. Hygrocybe miniata, $H$. rubrolamellata, $H$. calciphila, $H$. turunda, $H$. cantharellus, $H$. biminiata, $H$. substrangulata s.l., incl. $H$. substrangulata forma and H. substrangulata var. rhodophylla. Hygrocybe rubrolamellata is new to Europe, and Hygrocybe substrangulata var. rhodophylla is new to N. Europe. The taxonomic features of $H$. substrangulata s.1., $H$. substrangulata var. rhodophylla and $H$. biminiata are discussed.
\end{abstract}

Key words: Hygrocybe, Basidiomycetes, taxonomy, Arctic, montane, arctic-alpine mycology

Torbjørn Borgen, Sensommervej 142, 130, DK-8600, Silkeborg, Denmark; e-mail: torbjoern.borgen@gmail.com

Esteri Ohenoja, Botanical Museum, University of Oulu, P.O. Box 3000, FI-90014 University of Oulu, Finland; e-mail: esteri.ohenoja@oulu.fi

\section{Introduction}

The aim of the present investigation was to study a number of collections within Hygrocybe subsect. Squamulosae from mainly (sub)arctic and montane sites. The field studies motivated us to make a closer study of Hygrocybe biminiata Kühner and $H$. substrangulata (P.D. Orton) P.D. Orton \& Watling (s.l.) and their relations. H. biminiata has been regarded as a doubtful species by Boertmann (2010), but we are convinced that it deserves taxonomic recognition, in accordance with Kühner (1976) and Borgen \& Arnolds (2004). A lot of effort was allocated to the study of the highly variable $H$. substrangulata including the study of additional material, and the use of molecular methods. This article is part of the proceedings of the $9^{\text {th }}$ International Symposium of Arctic and Alpine Mycology (ISAM) held at Kevo Subarctic Research Station in Inari Lapland, Finland, 26.08. - 01.09.2012.

\section{Material and methods}

In this article we follow Boertmann (2010) in delimiting subsection Squamulosae. Most of the specimens studied are from N. Norway (mainly Finnmark and Troms) and N. Finland (Inari Lapland). Collections from more southern, boreal sites are only exceptionally included. A small number of collections are from arctic Canada: (N.W.T., District of Keewatin, Rankin Inlet $\left(62^{\circ} 48-51^{\prime} \mathrm{N}, 92^{\circ}\right.$ $\left.05-09^{\prime} \mathrm{W}\right)$, and Baker Lake (64. $\left.18^{\prime} \mathrm{N}, 96^{\circ} 08^{\prime} \mathrm{W}\right)$. Collections from Russia: (Tjumenskaja Oblast, S-Jamal $\left(67^{\circ}\right.$ $\left.30^{\prime} \mathrm{N}, 70^{\circ} 00^{\prime} \mathrm{E}\right)$, Labytnangi area $\left(67^{\circ} 01^{\prime} \mathrm{N}, 66^{\circ} 40^{\prime} \mathrm{E}\right)$, and the coast of the Kara Sea $\left(68^{\circ} 30^{\prime} \mathrm{N}, 67^{\circ} 00^{\prime} \mathrm{E}\right)$ are 


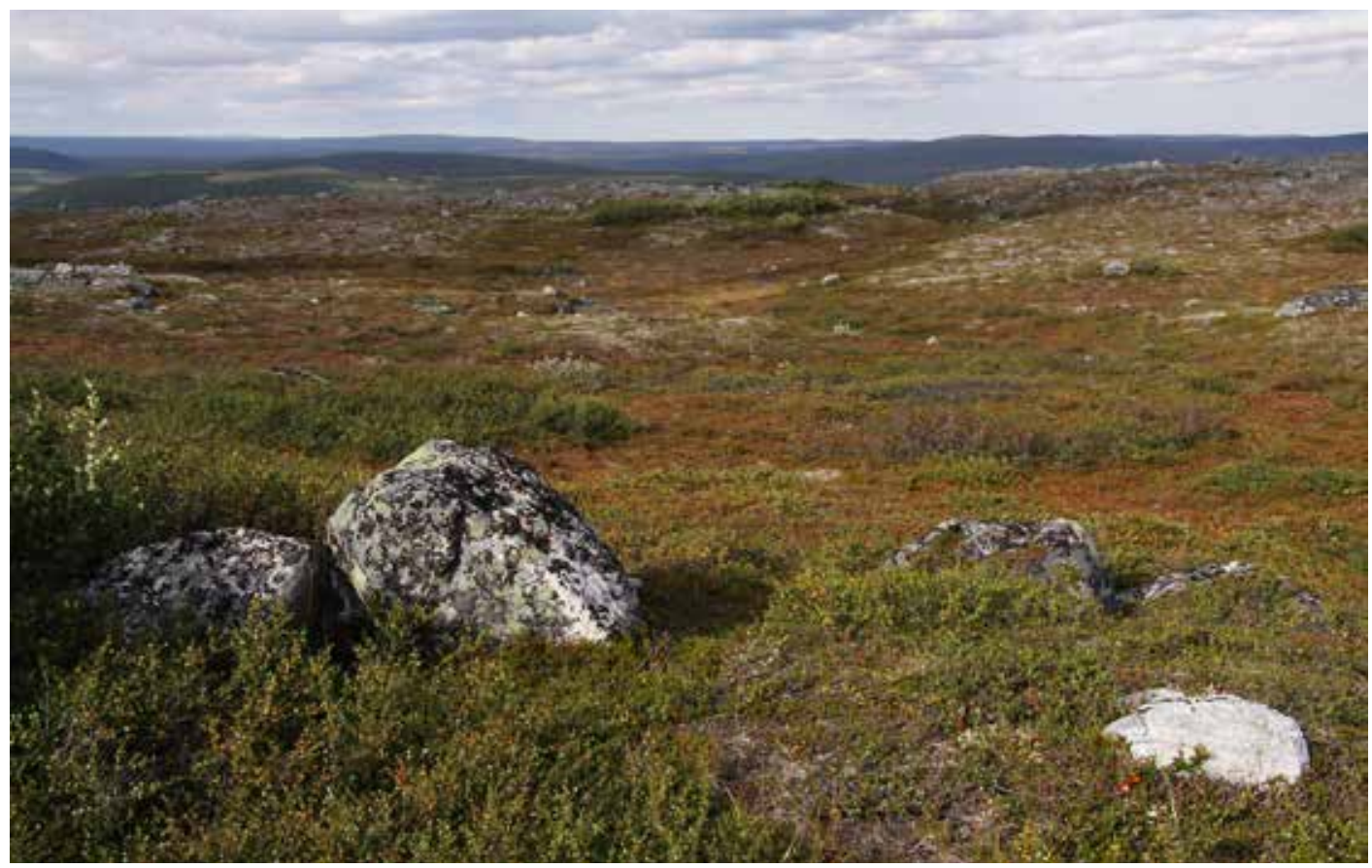

Fig. 1. Arctic heath. Utsjoki, Ailegas, 27.VIII.2012. Photo A. Ronikier

also included. A part of the material was collected during the Fifth International Symposium on Arctic-Alpine Mycology (ISAM 5) in the year 1996 (Russia). A number of fresh collections were made from Finland and Norway during ISAM 9 in the year 2012 (Figs. 1-4).

The studied collections are deposited in the following herbaria: Copenhagen (C), Krakow (KRAM), Oulu (OULU), Oslo (O), Trondheim (TRH), Tromsø (TROM) and Turku (TUR) and some collections are stored in the private herbarium of T. Borgen (TB), but they will be transferred later to $\mathrm{C}$. The collection quality varies. When they are well dried, ample, and supplied with photos and/ or field notes they are representative. Those collections, on which the descriptions are based, are cited with their herbarium numbers, those that are further discussed in the text are indicated with an asterisk in the list of specimens studied. Many of the remaining collections were also closely studied, while others were just checked microscopically to secure identification. In all 80 collections were studied. Approximately 20 of these could not be identified or belonged to other sections. Most emphasis has been given to the material from arctic Canada, Inari Lapland and Finnmark, because a lot of material from there was seen and studied in a fresh state. A few earlier published, but now re-identified collections are mentioned under each species.

A small number of photos of $H$. biminiata and $H$. substrangulata (including a deviating form, and var. rhodophylla) was available for further documentation (Figs. 6a- f). Drawings of terminal elements of pileipellis and stipe "hairs" are given based on microphotos of these species, and $H$. rubrolamellata. Drawings of the stipititrama of $H$. biminiata and $H$. cantharellus (Schwein.:Fr.) Murrill are included for comparison. Drawings of spores are included for all species (Figs. 5a-i).

When studying the material from the subsection Squamulosae our pre-assumption was that the following macroand microscopic features would be especially important, partly based on Borgen \& Arnolds (2004): presence/absence of dark pileal scales, stipe length/pileus width in mature specimens, gill attachment, colours of pileus and lamellae. The last two features were considered important especially concerning $H$. biminiata, $H$. substrangulata s.l. (incl. var. rhodophylla) and H. rubrolamellata T. Borgen $\&$ Arnolds. These features were also estimated from dried material (seemingly useful in some cases but not stated very explicitly in Borgen \& Arnolds (2004). Fresh material of $H$. biminiata, $H$. substrangulata, including var. rhodophylla (during ISAM 9) and $H$. miniata (Fr.: Fr.) P. Kumm. (Danish, checked material) were examined for eventual differences in stipe-covering using stereoscopic microscopes.

Average sizes of the terminal elements (especially length) of the scales in the pileipellis, in optimal sections were measured from one or two scales. It was also noted if the terminal elements were strikingly constricted in the midportion and at septa, and if narrow elements were present, or not. During ISAM 9 all fresh collections were quickly 


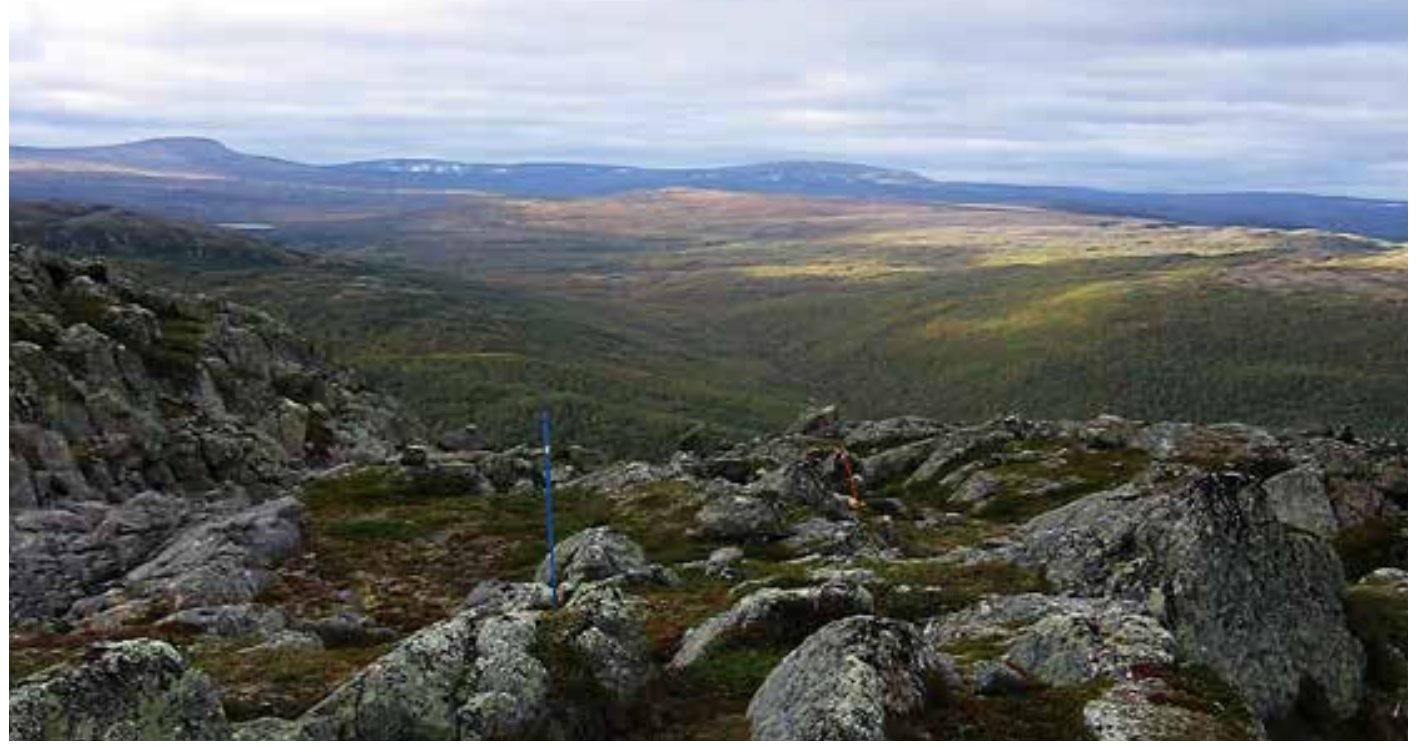

Fig. 2. Arctic heath on calcareous ground. Utsjoki, Gistuskaidi, 24.VIII.2011. Photo A. Ruotsalainen

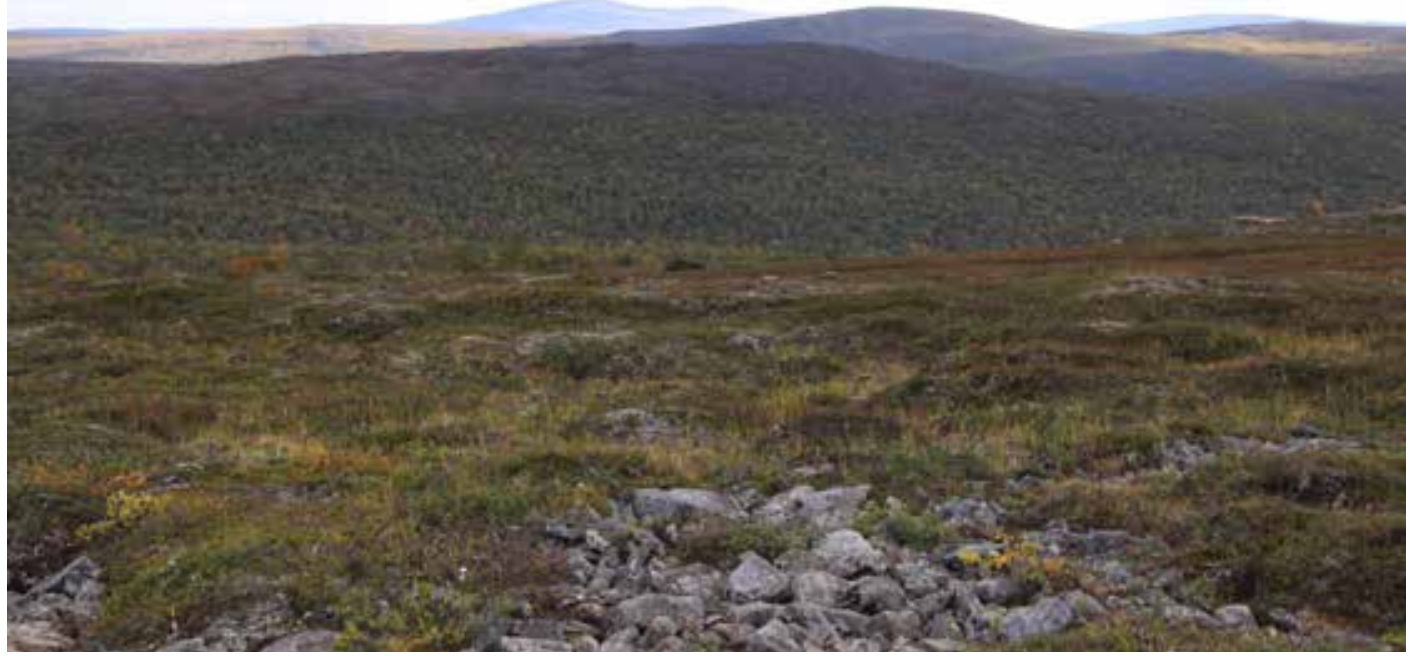

Fig. 3. Hummocky arctic heath. Utsjoki, Nuvvus Áilegas, 30.VIII.2012. Photo A. Ronikier 


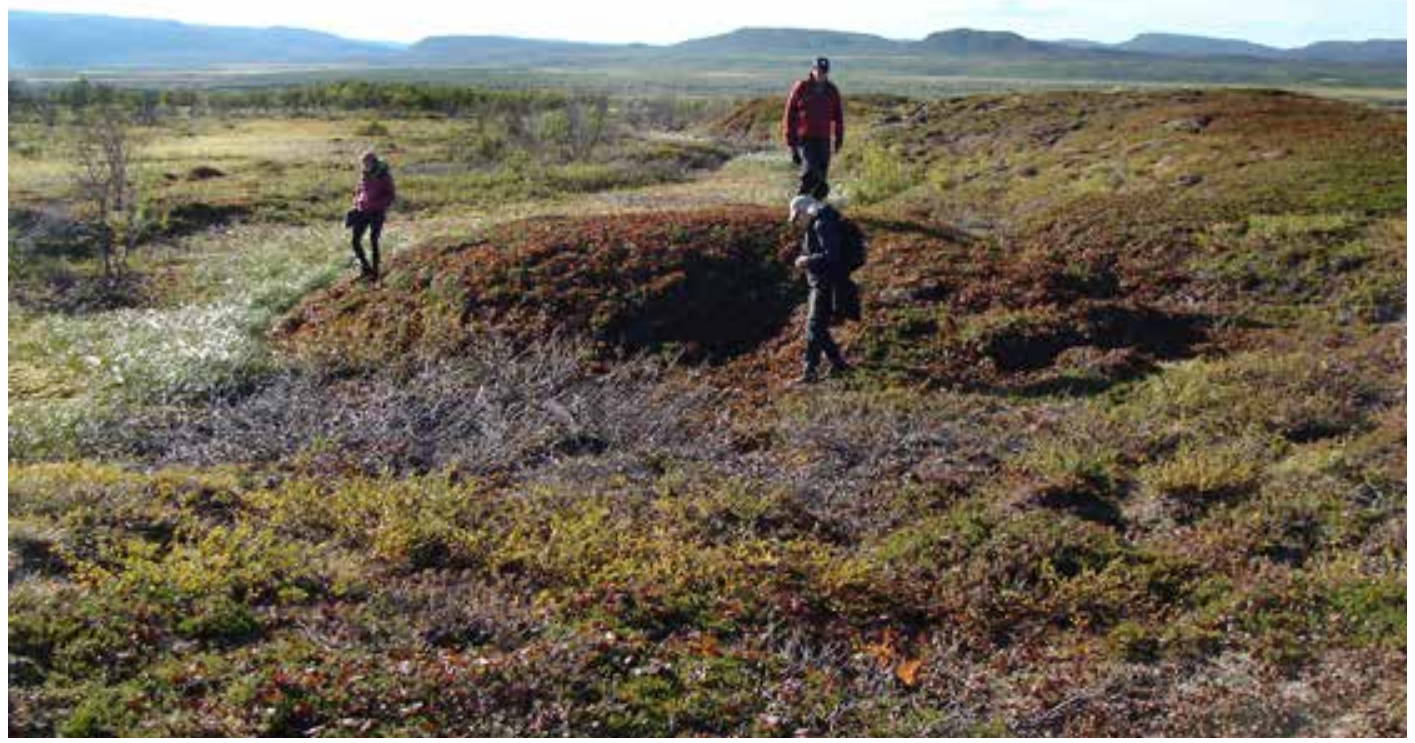

Fig. 4. Palsa bog at Karlebotn (Norway, Finnmark) 29.VIII.2012. Photo E. Ohenoja

tested for eventual colour reactions on the pileus with 10 $\% \mathrm{KOH}$ (a recommendation from P.-A. Moreau used by him in an unpublished study from the French Jura). The average width of the terminal elements in the stipitipellis was studied from those occurring in groups, bundles or fascicles. A few "free" hyphae were also included when these were more or less perpendicular to the orientation of the stipitipellis hyphae (cf. Borgen \& Arnolds 2004 for a closer characterization). Only stipe "hairs" with a basal septum were studied. Those lacking a septum ("proliferations") were noted when present, but not treated systematically.

In Borgen \& Arnolds (2004) the stipititrama elements were characterized as follows, relating to a length of 135 $\mu \mathrm{m}$ : shorter (in $H$. miniata, $H$. biminiata, $H$. substrangulata s.l. and $H$. rubrolamellata (called the miniata-group) or longer (in $H$. cantharellus, H. coccineocrenata and $H$. turunda, called the turunda-group). Exact measurements and averages are, however, only exceptionally achieved (but at least in one collection per species) in the present work. Based on observations, initially by P.-A. Moreau and later expanded by Borgen \& Arnolds (2004), it seemed that the miniata-group had stipe hairs, while these were absent in the turunda-group. Spore measurements were measured using accumulations on the stipe apex or from pileus surfaces. The average spore size, and (almost) absence $(<20 \%)$ or frequent presence of constriction $(>20 \%)$, as well as shape, were also judged to have some importance (cf. Table 2). All averages are based on at least ten measurements per collection, unless stated otherwise.

Techniques: revival of bits of deficient, weathered and old herbarium material, soaked in $8 \%$ aqueous ammonia (with or without Congo-red) or $3 \% \mathrm{KOH}$, succeeded well if kept from $\sim 0.5$ hour up to 1-2 days. This was especially important when studying the terminal elements of the pileipellis and the stipe hairs. Otherwise the material was treated like in Borgen \& Arnolds (2004). In that article, the number of nuclei in the spores was established in some cases; however, we have not attempted this here, as the results would not seem to be relevant for the separation of $H$. biminiata and $H$. substrangulata s.l. Microscopic investigation was made using an Olympus $\mathrm{CH} 2$ microscope, and drawings are based on photos obtained by replacing an eyepiece with a tube-extention connected to a Coolpix 4500 camera. Spores were photographed at $\times 1000$ using the immersion lens (N.a. 1.30); other structures were photographed at $\times 400$, or $\times 600$. The numbers of entire (L) and short (1) lamellae are occasionally indicated. Colours are coded using Kornerup \& Wanscher (1978), unless stated otherwise. 
Key (mainly based on representative collections and on the information of Borgen \& Arnolds 2004).

1A: Stipititrama consisting of large elements, mainly $>200 \mu \mathrm{m}$ long, stipitipellis without hairs. Lamellae always (sub)decurrent.

1B: Stipititrama made up of short elements, $<125 \mu \mathrm{m}$ long, stipitipellis mostly with single and groups of "hairs". Lamellae decurrent or not

2A: Pileus with minute, (sub)concolorous scales Hygrocybe cantharellus (p. xxx)

2B: Pileus with minute to coarse, brownish to blackish scales

3A: Pileus with distinct to coarse, predominantly brown to blackish scales on a scarlet to orange-yellow background. Lamellae pale to deep yellow, sometimes orange-yellow. Mostly in mesic to dry grassland slopes H. turunda (p. yyy)

3B: Pileus with minute, usually dark brown to blackish scales on red to orange-red background. Lamellae white, then cream-coloured to wax yellow, occasionally pale red near base.

Mostly in Sphagnum bogs, never in grassland. Absent or rare beyond the boreal area in

N. Scandinavia, but included in the key due to confusion with slender collections of

H. substrangulata s.1

H. coccineocrenata

4A: Spores up to $9 \mu \mathrm{m}$ long on average, broader towards apiculus and frequently constricted, or ellipsoid, ovoid to subphaseoliform. Pileus with concolorous scales. Rare or overlooked species, at least in the montane and subarctic habitats.

4B: Spores on average 9-11.5 $\mu \mathrm{m}$ long at most. Frequent in N. Scandinavia. 7

5A: Spores in frontal view broader towards apiculus and often constricted, obovoid to obpyriform, in side view ellipsoid. Usually on acid soils..... H. miniata (p. zzz)

5A: Spores (shortly) ellipsoid to ovoid, not constricted 6

6A: Pileus generally orange, lamellae usually yellowish. Spore quotient $\leq 1.5$, not constricted, stipe exceptionally with hairs, which are on average $5.6 \mu \mathrm{m}$ wide.

On calcareous soils H. calciphila (p. aaa)

6B: Pileus and lamellae red. Spores narrower, on average $9.1 \times 5.3 \mu \mathrm{m}$. Stipe hairs on average $3.9 \mu \mathrm{m}$ wide $H$. rubrolamellata (p. bbb)

\section{THE SPECIES}

Hygrocybe miniata (Fr.: Fr.) P. Kumm. - Fig. 5a, Table 2

Spores on average $8.7 \times 6.0 \mu \mathrm{m}, \mathrm{Q}_{\mathrm{m}}=1.46$, in side view $10 \%$, in frontal view $50 \%$ constricted, ellipsoid, ovoid to obpyriform. Basidia predominantly 2 -spored. Hymenophoral trama of short, subcylindric elements. Pileipellis a trichoderm, terminal elements $(10 / 1)$ on average $55.4 \times 8.4$ $\mu \mathrm{m}$. Stipitipellis a dry cutis, with scattered bundles of very entangled, narrow hairs $(6 / 1)$, on average $27.0 \times 3.6 \mu \mathrm{m}$. Stipititrama consisting of radial short, mainly subcylindric elements $(10 / 1)$, on average $85.9 \times 13.6 \mu \mathrm{m}$. Clamp connections not observed.
Habitat: No information.

Specimen studied: NORWAY. Finnmark. Polmak, 22.VIII.1961 Kallio (TUR 20113).

The material consists of three specimens in a fairly good state. The collection was filed as $H$. cantharellus, there are no further notes on the label.

\section{Hygrocybe rubrolamellata T. Borgen \& Arnolds} - Figs. 5b, j, Table 2

According to the original notes the pileus was less than $10 \mathrm{~mm}$, scarlet and the stipe scarlet in the upper part (the remaining part of the infor- 


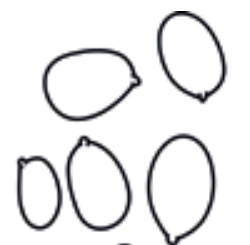

a

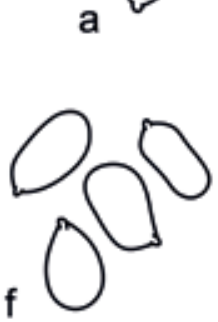

f

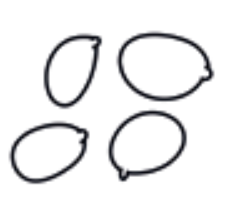

c
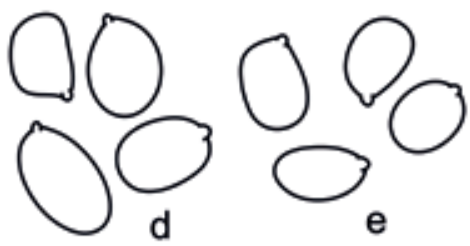

b
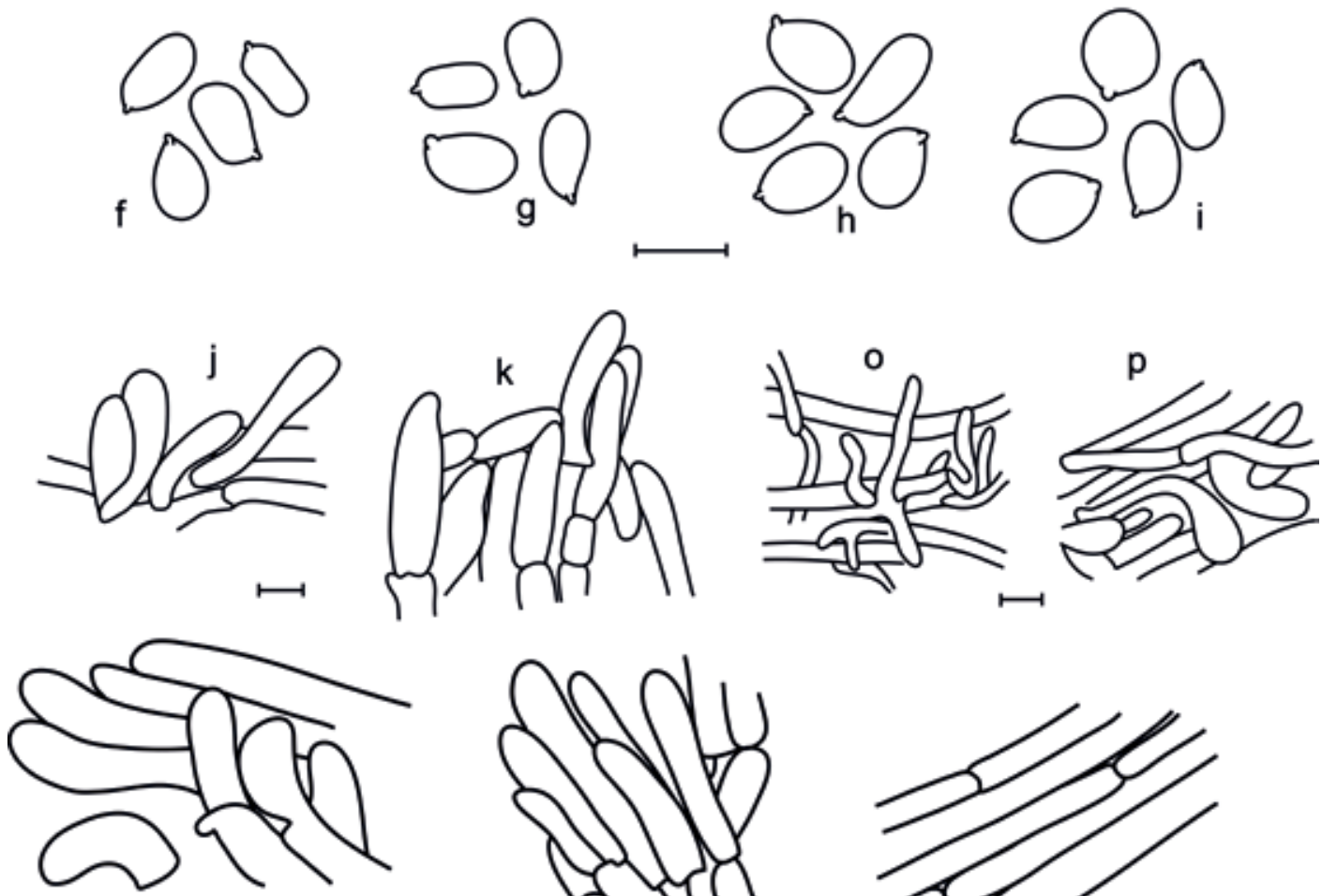

I
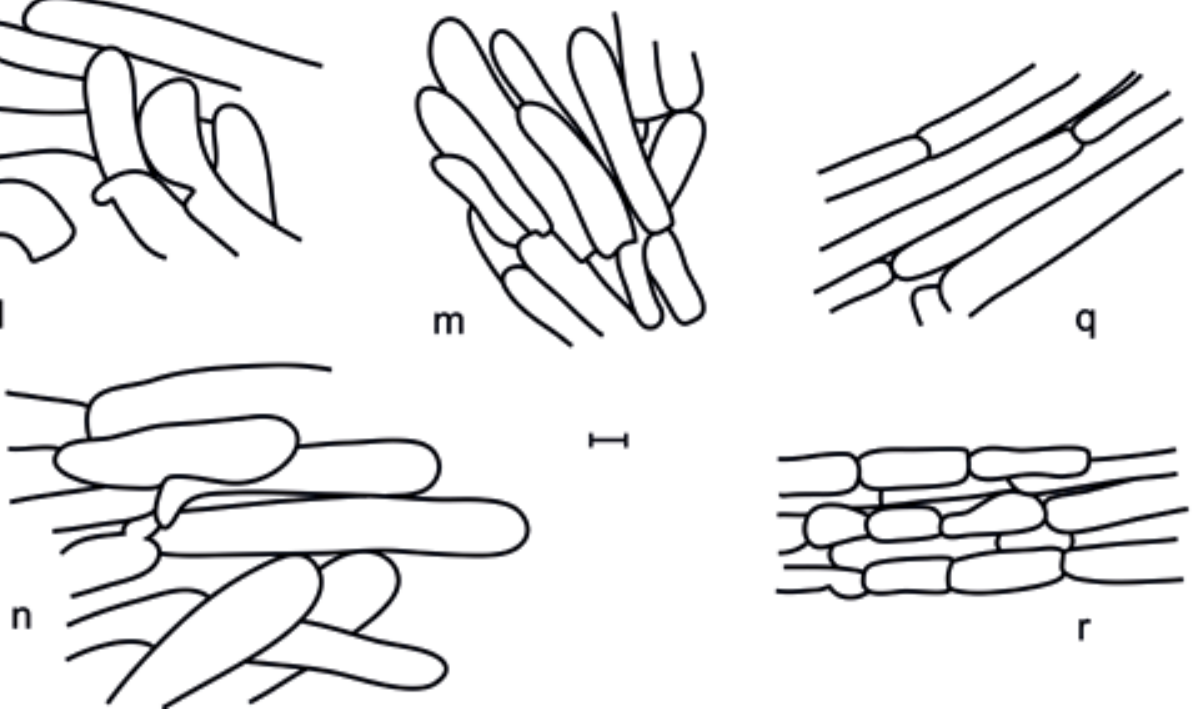

Fig. 5. Microscopic elements of arctic-alpine Hygrocybe spp.; spores (a-i), pileipellis (j-n), stipe hairs (o, p), stipititrama $(\mathrm{q}, \mathrm{r}) . \mathrm{a}=H$. miniata $($ TUR 20113) $-\mathrm{b}, \mathrm{j}=$ H. rubrolamellata $($ TROM F-22716) $-\mathrm{c}=H$. calciphila $($ TROM $\mathrm{F}-31605)-$ $\mathrm{d}=$ H. turunda $($ TROM F-12502) $-\mathrm{e}, \mathrm{q}=$ H. cantharellus $($ TROM F-9143) $-\mathrm{f}, \mathrm{k}, \mathrm{o}, \mathrm{r}=$ H. biminiata $($ TB 12.008) $-\mathrm{g}$, $1, \mathrm{p}=H$. substrangulata var. substrangulata (KRAM F-49441 / TB 12.045) $-\mathrm{h}, \mathrm{m}=H$. substrangulata var. substrangulata forma (TB 12.042) $-\mathrm{i}, \mathrm{n}=$ H. substrangulata var. rhodophylla. Scales $=10 \mu \mathrm{m}$. 
mation was unreadable). Spores (16/1) on average $9.1 \times 5.3 \mu \mathrm{m}$, predominantly ellipsoid, not constricted. $Q_{m}=1.72$. Only basidioles seen. Hymenophoral trama subregular, consisting of short elements. Pileipellis, terminal elements (12/1) on average $42.8 \times 6.6 \mu \mathrm{m}$, hyaline in aqueous ammonia, subcylindrical, less frequently subclavate to subfusiform. Stipitipellis dry, in places a less compact cutis, stipe hairs in entangled groups, hairs mostly irregularly flexuose and undulate $(12 / 1)$, on average $35.0 \times 3.9 \mu \mathrm{m}$, proliferations present. Stipititrama elements short, (sub)cylindrical $(9 / 1)$, on average $62.5 \times 11.4 \mu \mathrm{m}$. Oleiferous hyphae present. Clamp connections present.

Habitat: At the forest limit, in tight moss vegetation.

Specimen studied: NORWAY. Troms. Gratangen, Helleshamn, NE slope of Skavlikollen, 14.VIII.1993 Engelskjøn \& Skifte (TROM F-22716).

Hygrocybe rubrolamellata has so far been recorded only from subarctic S. Greenland (Borgen $\&$ Arnolds 2004). The dried material consists of four very dark, partly ripe specimens in a fairly good state. The spores are slightly larger than in the diagnosis of $H$. rubrolamellata (average 9.1 $\times 5.3 \mu \mathrm{m}$, versus $7.2-8.4 \times 4.2-5.1 \mu \mathrm{m}$ ), while the terminal elements of the pileipellis are largely of the same size $(42.8 \times 6.6 \mu \mathrm{m}$ versus $39-40$ $\times 6.5-8.5 \mu \mathrm{m})$. The dry material is very similar to the holotype. The spore size was thought to be different enough from $H$. biminiata (cf. Borgen \& Arnolds 2004, fig. 19) to constitute an additional character, but this is not the case. However, the colours are very different, also in dry material. The collection was filed as $H$. miniata.

\section{Hygrocybe calciphila Arnolds}

- Fig 5c, Table 2

Spores $(12 / 1)$ on average $9.1 \times 6.0 \mu \mathrm{m}$, (subglobose) ovoid to ellipsoid, not constricted. $\mathrm{Q}_{\mathrm{m}}=1.50$. Basidia 4-spored, cystidia not observed, hymenophoral trama subradial, elements short, cylindrical to inflated; pileipellis terminal elements $(10 / 1)$ on average $63.7 \times 9.5 \mu \mathrm{m}$. Stipitipellis a dry cutis, stipe hairs single or in groups $(10 / 1)$, on average $49.5 \times 5.6 \mu \mathrm{m}$; proliferations present. Stipititrama subregular, elements $(10 / 1)$ on average $81.8 \times 10.6 \mu \mathrm{m}$, predominantly subcylindrical, occasionally attenuated, clamp connections present.

Specimen studied: NORWAY. Nordland. Steigen, SW. side of Jamtfjell, 12.VIII.1966 Skifte \& Engelskjøn (TROM F-31605)

Habitat: Above forest limit in grass field on calcareous soil.

The material consists of four or five, partly fragmented specimens in a mediocre state; no field notes were present. The spores are slightly larger than in the the diagnosis. However, similar measurements were given by Borgen \& Senn-Irlet (1995). The presence of stipe hairs is discussed further below. The collection was filed as Hygrophorus miniatus.

Hygrocybe turunda (Fr.: Fr.) P. Karst. sensu F.H. Møller, P.D. Orton (1960 in part) - Fig. 5d, Table 2

The brief, partly legible field notes are: "pileus $35 \mathrm{~mm}$, dark squamulose, over orange. Lamellae strongly decurrent, distant, yellowish orange with olivaceous tinge. Stipe $45 \times 2.8-3.4 \mathrm{~mm}$, fistulose, flexuose, glabrous. Context yellow". Spores (16/1) on average $9.4 \times 6.1 \mu \mathrm{m}, \mathrm{Q}_{\mathrm{m}}=1.54$, in side view $0 \%$, in frontal view $5 \%$ constricted, predominantly ellipsoid. Basidia 4-spored, edge fertile, cystidia not seen. Hymenophoral trama consists of short elements. Pileipellis a trichoderm, terminal elements (10/1) on average 54.3 $\times 12.5 \mu \mathrm{m}$, elements subcylindrical, subattenuated, clavate, lanceolate to subfusiform, with pale grey-brown, partly dotted pigment. Stipitipellis a dry cutis, without genuine hairs, merely few ascending hyphal ends. Stipititrama subregular, elements $(10 / 1)$ on average $221.3 \times 20.9 \mu \mathrm{m}$, predominantly (partially) attenuated to subcylindrical.

Habitat: Grassy ground with e.g. Equisetum arvense, Vaccinium myrtillus, Empetrum and Alchemilla alpina. 
Specimens studied: NORWAY. Troms. Troms $\varnothing$, Tromsdalen, down from Dalheim on the N. side of the river, alt. $\sim 400 \mathrm{~m}, 23$.VIII.1969 Skifte 112/69 (TROM F-12502); Lyngen, Kvalvikdalen at Tverrelva, 30.VII.1983 Sivertsen \& L.Sivertsen (TRH); below Oksefjellet, 7.VIII.1977 Sivertsen 77-40 (TRH).

The first collection (filed as $H$. cantharellus) consists of two slightly fragmented specimens in a mediocre state (scales collapsed, leaving a dark surface). The two latter collections, being in good state, were identified, but not included in the description.

Hygrocybe cantharellus (Schwein.: Fr.) Murrill - Figs. 5e, q, Table 2

The brief field notes are: "Lamellae distinctly decurrent". Spores $(12 / 1)$ on average $8.7 \times 6.0 \mu \mathrm{m}$, $\mathrm{Q}_{\mathrm{m}}=1.46$, not constricted. Basidia predominantly 4-spored (a few 2-spored). Hymenophoral trama made up of (fairly) short elements, cylindrical to inflated, some irregular. Pileipellis a trichoderm, only two elements could be measured, viz. 51$76 \times 12-14.5 \mu \mathrm{m}$. Stipitipellis a dry cutis, hairs absent. Stipititrama, regular made up of (fairly) long elements $(10 / 1)$ on average $262.3 \times 14.8$ $\mu \mathrm{m}$, subcylindrical to sublanceolate. Clamp connections present.

\section{Habitat: Among mosses.}

Specimen studied: NORWAY. Troms. Balsfjord, E-side of Balfjord, $1 \mathrm{~km}$ S of Tromsø hd. Storkallan-Tuva, N-WNW slope above the road, alt. $\sim 100 \mathrm{~m}, 12 . \mathrm{IX} .1982$ A.Skifte OS252/82 (TROM F-9143).

The material is in a bad state and somewhat broken but still identifiable.

\section{Hygrocybe biminiata Kühner} - Figs. 5f, k, o, r, 6a, b, Tables 1, 3

Misapplied names:

Hygrophorus marchii ss. Kallio \& Kankainen, in part $(1964,194)$
?Hygrophorus miniatus var. sphagnophilus Peck ss. Lange \& Skifte (1967: 15).

Pileus 20-25 mm wide, convex, plano-convex to subumbonate, later plano-convex with a depressed centre, orange to reddish orange about $8 \mathrm{~A} 7$ to $8 \mathrm{~A} 8$, in younger specimen about 7A7; inwards "duller", more watery and diffusely coloured, fibrillose-tomentose to squamulose, scales yellowish, concolorous to slightly darker, surface immediately hardly changing in $10 \% \mathrm{KOH}$, then slightly fading. Lamellae adnate to emarginated with or without tooth, subdistant, $(\mathrm{L}=20-25$, $1=0-3$ ) fairly thick, slightly ventricose, up to 5 $\mathrm{mm}$ broad; yellow to bright yellow (4A5 with suggestion of $5 \mathrm{~A} 5$, or $4 \mathrm{~A} 6-7)$, then with a reddish tinge. Stipe up to $35-45 \times 4-7.5 \mathrm{~mm}$, subcylindric to attenuated, fistulose, dry, dull to slightly shiny, apical part almost concolorous with the pileus, or paler ( $\sim 8 \mathrm{~A} 7$ to $7 \mathrm{~A} 6)$, yellowish below $(\sim 4 \mathrm{~A} 7)$, downwards yellowish $(\sim$ 4A7-4A6) to whitish; almost glabrous, at most slightly rugulose in the apical part. Context (sub) concolorous, when older with cavity in stipe, not brittle, smell none or slight, taste mild. Spores on average 9.0-9.8 × 5.2-6.1 $\mu \mathrm{m}, \mathrm{Q}_{\mathrm{m}}=1.60-1.77$, ellipsoid to oblong, occasionally ovoid, in side view $10-30 \%$, in frontal view $40-45 \%$ constricted. Basidia 4-spored, 10/1: Q $=(4.1-) 4.8-7.2$, predominantly subclavate. Edge fertile, with scattered, basidioles or pseudocystidia, irregular, some even almost subcoralloid, $\sim 26-37 \times$ 3.5-5.5 $\mu \mathrm{m}$. Hymenophoral trama subregular, made up of short, predominantly (sub)cylindrical elements. Pileipellis a trichoderm, terminal elements on average $36.1-39.5 \times 7.0-9.6 \mu \mathrm{m}$, elements predominantly (sub)cylindrical, occasionally attenuated, rarely clavate, pale ochre-yellow at low magnification, hardly constricted at septa, narrow hyphae not seen; pileitrama not studied. Stipe hairs in groups, in fascicles or occasionally single, on average $25-45 \times 4.4-5.0 \mu \mathrm{m}$, flexuose; proliferations present. Stipititrama subregular, elements predominantly subcylindrical on average $64.0 \times 13.8 \mu \mathrm{m}$. Clamp connections present.

Habitat: In mesic to (dry) dwarf scrub heaths, or in drier part of (palsa) bogs but not in Sphagnum; with e.g. Betula nana, Empetrum, Vaccinium uliginosum and V. vitis-idaea; a Canadian record in depression on dry Dryas-Cassiope heath with Salix reticulata. 

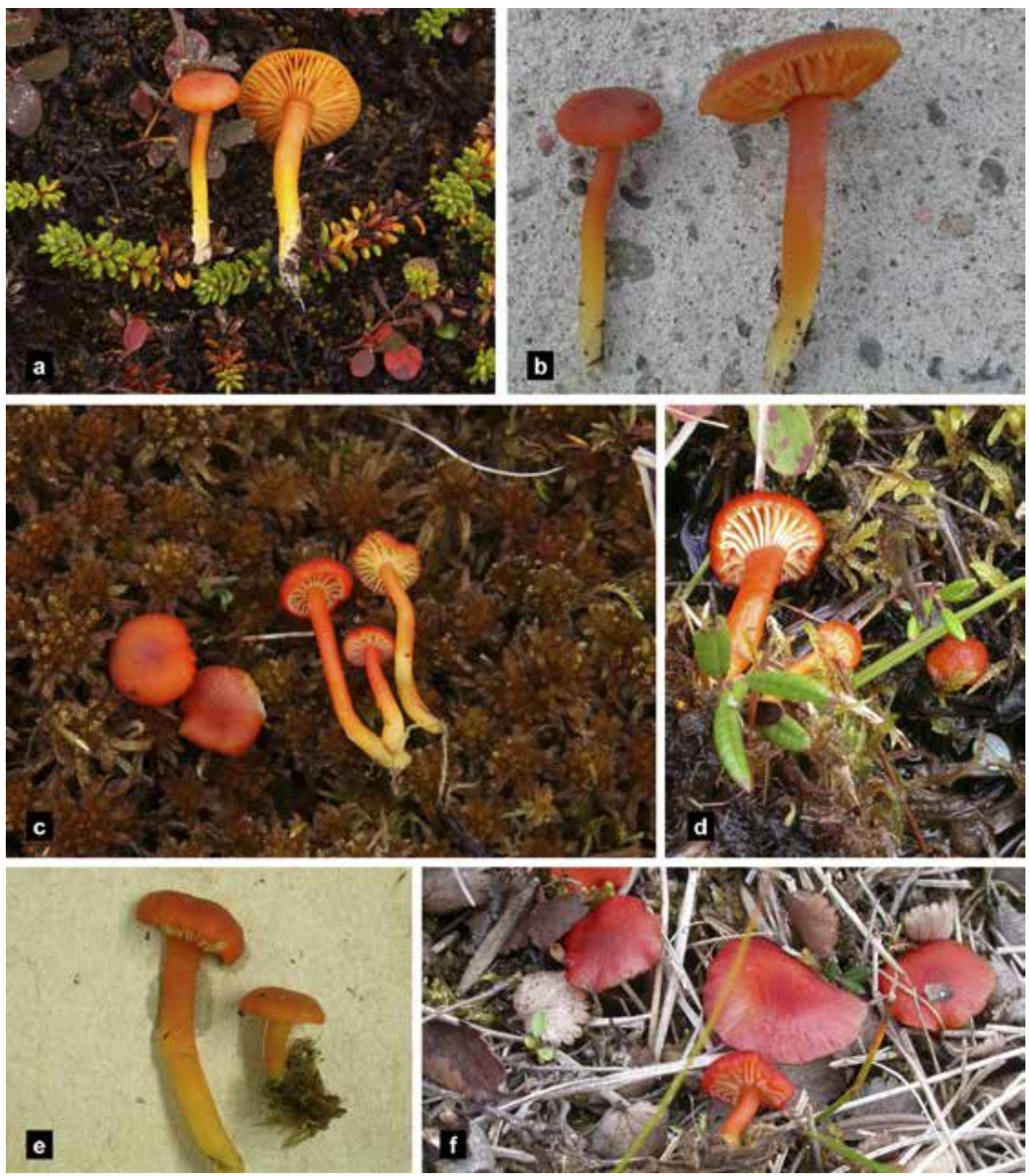

Fig. 6. Fresh fruitbodies of arctic-alpine Hygrocybe spp. $\mathrm{a}-\mathrm{b}=\mathrm{H}$. biminiata $(\mathrm{TB} 12.008$. Photo A. Ronikier; b. Photo T. Borgen) $-\mathrm{c}=$ H. substrangulata var. substrangulata (KRAM F-49441. Photo A. Ronikier) $-\mathrm{d}$, e left $=$ Hygrocybe substrangulata var. substrangulata forma (TB 12.042. Photo T. Borgen) $-\mathrm{e}$ right $=$ Hygrocybe substrangulata var. substrangulata (TB 12.043. Photo T. Borgen) $-\mathrm{f}=$ Hygrocybe substrangulata var. rhodophylla (TB 12.044. Photo T. Borgen). 

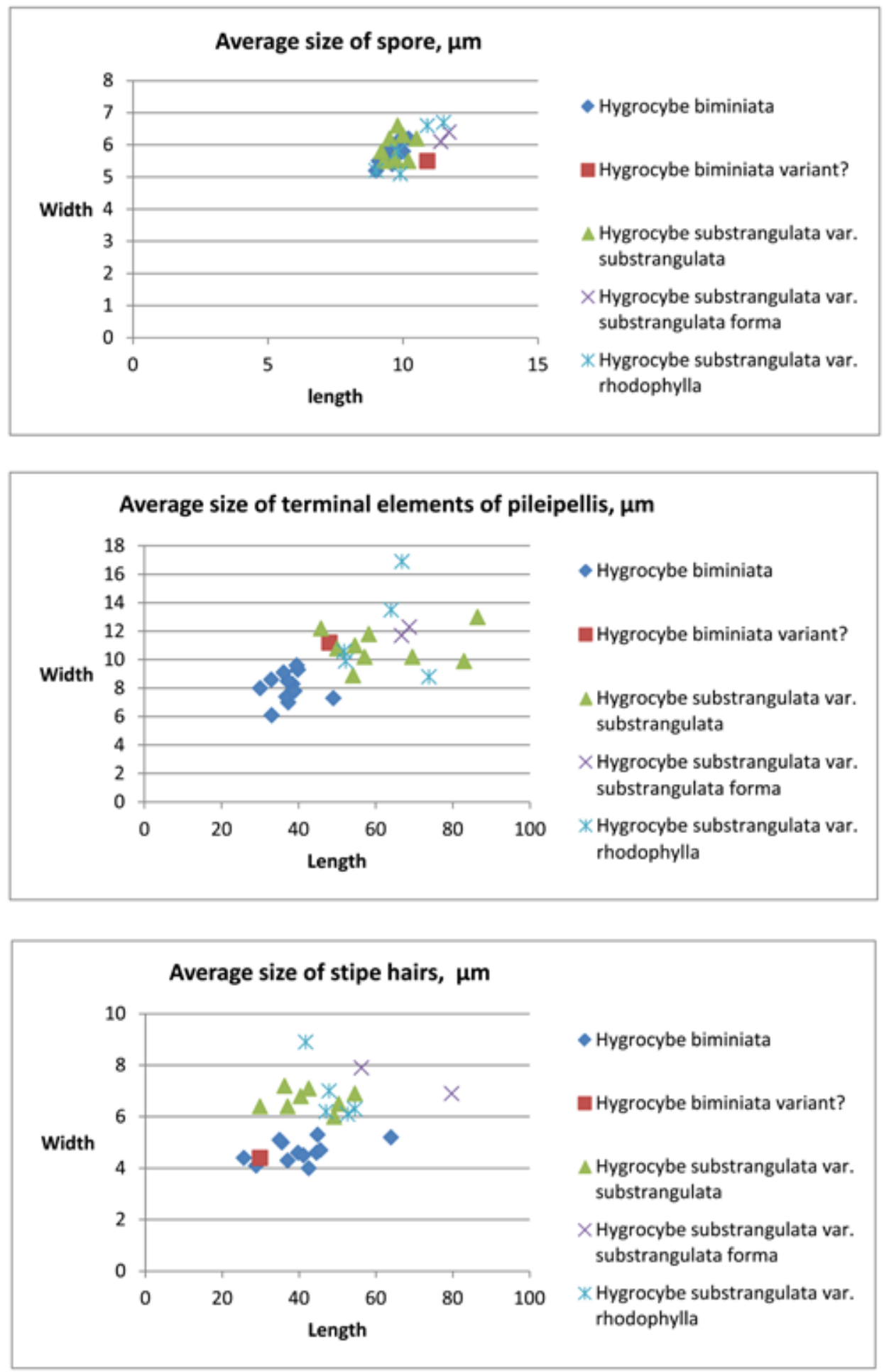

Fig 7. Populational averages of microscopic elements in Hygrocybe subsect. Squamulosae. Averages are based on the measurements of ten cells from each population. 


\begin{tabular}{|c|c|c|c|c|c|c|c|c|c|c|c|c|c|c|}
\hline Epithet & Herb. & Coll. No & $\begin{array}{c}\text { Spore } \\
\text { L }\end{array}$ & $\begin{array}{c}\text { Spore } \\
\text { W }\end{array}$ & Qm & $\underset{\text { constr }}{P}$ & $\begin{array}{c}V \\
\text { constr }\end{array}$ & $\begin{array}{l}\text { Pileus } \\
\text { E L }\end{array}$ & $\begin{array}{c}\text { Pileus } \\
\text { E W }\end{array}$ & $\begin{array}{c}\text { Stipe } \\
\text { H L }\end{array}$ & $\begin{array}{l}\text { Stipe } \\
\text { H W }\end{array}$ & $\begin{array}{c}\text { Stipe } \\
\text { T L }\end{array}$ & $\begin{array}{l}\text { Stipe } \\
\text { T W }\end{array}$ & $\begin{array}{l}\text { feature } \\
\text { analysis }\end{array}$ \\
\hline Hygbim & TROM & T 31590 & 9,7 & 5,8 & 1,76 & 20 & 35 & 39,8 & 9,3 & 44,5 & 4,6 & & & \\
\hline Hygbim & $\mathrm{TB}$ & TB 12.008 & 9,8 & 6,1 & $1,6,0$ & 10 & 40 & 38,4 & 8,3 & 25,6 & 4,4 & 64,0 & 13,8 & Xýdl \\
\hline Hygbim & TB & TB 12.029 & 9,0 & 5,2 & 1,72 & 10 & 46 & 39,5 & 9,6 & 45,5 & 4,7 & & & Oydl \\
\hline Hygbim & OULU & F343339 & 9,4 & 5,5 & 1,72 & 35 & 37 & 37,2 & 8,5 & 39,7 & 4,6 & & & Xýdl \\
\hline Hygbim & OULU & F042562 & 9,7 & 5,6 & 1,71 & 33 & 35 & 32,9 & 8,6 & 63,9 & 5,2 & & & Xodl \\
\hline Hygbim & OULU & F073587 & 9,6 & 5,4 & 1,77 & 20 & 30 & 37,3 & 7,0 & 35,5 & 5,0 & & & Xydl \\
\hline Hygbim & OULU & F072841 & 9,1 & 5,5 & 1,61 & 0 & 20 & 36,1 & 9,1 & 28,8 & 4,1 & & & Xydl \\
\hline Hygbim & OULU & F073485 & 9,8 & 5,9 & 1,69 & 5 & 5 & 49,0 & 7,3 & 44,8 & 5,3 & & & Oydl \\
\hline Hygbim & OULU & F073500 & 9,2 & 5,6 & 1,64 & 22 & 30 & 33,0 & 6,1 & 34,9 & 5,1 & & & Rydl \\
\hline Hygbim & TROM & T 1841 & 10,2 & 6,2 & $1,7,0$ & 10 & 30 & 36,8 & 7,4 & 37,0 & 4,3 & & & Oýdl \\
\hline Hygbim & $\mathrm{TB}$ & TB 96.015 & 10,0 & 5,8 & 1,71 & 12 & 25 & 38,9 & 7,8 & 41,1 & 4,5 & & & Oydl \\
\hline Hygbim & G & Type & 9,4 & 5,8 & 1,63 & 9 & 9 & 30,0 & 8,0 & 42,5 & 4,0 & 88,8 & 13,3 & Oýdl \\
\hline Hygbim? & OULU & F042311 & 10,9 & 5,5 & 1,98 & 20 & 55 & 48,0 & 11,2 & 29,8 & 4,4 & 84,6 & 11,1 & OydL \\
\hline Hygsub & TROM & T 31545 & 10,5 & 6,2 & 1,68 & 15 & 15 & 82,9 & 9,9 & 50,3 & 6,5 & & & RoDL \\
\hline Hygsub & TROM & T 11722 & 10,2 & 5,5 & 1,85 & 20 & 33 & 69,5 & 10,2 & 49,1 & 6,0 & & & RoDl \\
\hline Hygsub & TB & TВ 12.043 & 9,7 & 5,5 & 1,75 & 10 & 50 & 50,0 & 10,8 & 40,4 & 6,8 & & & Oydl \\
\hline Hygsub & TB & TB 12.001 & 9,3 & 5,5 & 1,69 & 5 & 10 & 58,2 & 11,8 & 29,8 & 6,4 & & & XyDL \\
\hline Hygsub & $\mathrm{TB}$ & TB 12.041 & 9,2 & 5,8 & 1,60 & 4 & 8 & 54,1 & 8,9 & 42,5 & 7,1 & & & XyDI \\
\hline Hygsub & $\mathrm{TB}$ & TB 12.045 & 9,5 & 6,2 & 1,53 & 5 & 5 & 45,8 & 12,2 & 54,5 & 6,9 & 84,4 & 11,6 & RyDL \\
\hline Hygsub & KRAM & KRAM & 9,8 & 6,6 & 1,49 & 0 & 0 & 54,6 & 11,0 & 54,5 & 6,9 & & & RyDL \\
\hline Hygsub & $\mathrm{C}$ & C 34270 & 10,0 & 6,3 & 1,59 & 10 & 10 & 57,1 & 10,2 & 37,0 & 6,4 & & & RyDL \\
\hline Hygsub & TROM & T 31596 & 9,7 & 5,6 & 1,74 & 14 & 26 & 86,4 & 13,0 & 36,2 & 7,2 & & & RodL \\
\hline Hygsub f & TB & TB 12.042 & 11,7 & 6,4 & 1,82 & 14 & 5 & 68,7 & 12,3 & 56,2 & 7,9 & 86,0 & 10,3 & OyDL \\
\hline Hygsub f & TROM & T 31749 & 11,4 & 6,1 & 1,88 & 7 & 14 & 66,7 & 11,7 & 79,7 & 6,9 & & & OoDL \\
\hline Hygrho & $\mathrm{TB}$ & TB 12.044 & 10,9 & 6,6 & 1,65 & 0 & 10 & 51,8 & 10,6 & 47,8 & 7,0 & 84,4 & 11,6 & Rrdl \\
\hline Hygrho & TROM & T 31595 & 9,9 & 5,1 & 1,92 & 13 & 13 & 66,8 & 16,9 & 47,0 & 6,2 & 77,9 & 11,6 & $\mathrm{RrDl}$ \\
\hline Hygrho & TROM & T 31594 & 9,0 & 5,2 & 1,74 & 3 & 37 & 64,0 & 13,5 & 52,6 & 6,1 & & & $\mathrm{RrDl}$ \\
\hline Hygrho & OULU & F076316 & 9,8 & 5,8 & 1,68 & 0 & 5 & 52,2 & 9,9 & 41,7 & 8,9 & & & RodL \\
\hline Hygrho & TROM & T 8761 & 11,5 & 6,7 & 1,75 & 24 & 30 & 73,8 & 9,8 & 54,5 & 6,3 & & & Rxdl \\
\hline
\end{tabular}

Table 1. Collection-based summary of microscopic and macroscopic features in arctic-alpine Hygrocybe spp. Hygbim $=H$. biminiata $;$ Hygbim $=H$. biminiata variant; Hygsub $=H$. substrangulata var. substrangulata $;$ Hygsub $\mathrm{f}=H$. substrangulata var. substrangulata forma; Hygrho $=$ H. substrangulata var. rhodophylla. - Spore L = average spore length; Spore $\mathrm{W}=$ average spore width; $\mathrm{Q}_{\mathrm{m}}=$ average spore quotient; $\mathrm{P}$ constr = median constriction in side view; $\mathrm{V}$ constr = median constriction in frontal view; Pileus $\mathrm{E} \mathrm{L}=$ terminal elements of pileipellis average length; Pileus $\mathrm{E} W=$ terminal elements of pileipellis average width; Stipe $\mathrm{HL}=$ stipe hairs (terminal element) average length; Stipe $\mathrm{H} W=$ stipe hairs (terminal element) average width; Stipe $\mathrm{T} \mathrm{L}=$ stipititrama elements average length; Stipe $\mathrm{T} \mathrm{W}=$ stipititrama elements average width. - Feature analysis: $1^{\text {st }}$ capital letter $=$ predominant pileus colour $(\mathrm{R}=$ red, $\mathrm{X}=$ orange red to reddish orange, $\mathrm{O}=$ orange $) ; 2^{\text {nd }}$ letter $=$ predominant gill colour $(\mathrm{y}=$ (pale) yellow, $y=$ deep yellow, $\mathrm{o}=$ orange, $\mathrm{x}=$ orange-red, $\mathrm{r}=\mathrm{red}) ; 3^{\text {rd }}$ letter $=$ gill $(\mathrm{D}=$ decurrent, $\mathrm{d}=$ not decurrent $) ; 4^{\text {th }}$ letter $=$ habitus (slenderness $)(\mathrm{L}=$ stipe $/$ pileus quotient $\geq 2,1=$ stipe $/$ pileus quotient $<2$ ). 


\begin{tabular}{|c|c|c|c|c|c|}
\hline & H. miniata & H. rubrolamellata & H. calciphila & H. turunda & H. cantharellus \\
\hline \multicolumn{6}{|l|}{ Spores } \\
\hline Length & $(6.9-) 7.4 \times 10.1(-10.6)$ & $(7.8-) 8.1 \times 9.6(-10.3)$ & $(6.9-) 8.1 \times 10.8(-11.5)$ & $(7.4-) 8.3 \times 10.3(-10.5)$ & $(7.1-) 7.4 \times 9.3(-9.8)$ \\
\hline $\begin{array}{l}\text { Width } \\
\text { Average } \\
\text { quotient } Q_{m}\end{array}$ & $\begin{array}{l}(4.7-) 5.2 \times 6.4(-7.4) \\
1.40 \times 1.52\end{array}$ & $\begin{array}{l}(4.4-) 4.7 \times 6.4(-6.6) \\
(1.35-) 1.4 \times 1.85(-1.9)\end{array}$ & $\begin{array}{l}5.1 \times 6.4(-8.3) \\
(1.1-) 1.25 \times 1.65(-1.9)\end{array}$ & $\begin{array}{l}5.4 \times 6.6 \\
(1.25-) 1.4 \times 1.65(-1.70)\end{array}$ & $\begin{array}{l}(4.9-) 5.1 \times 6.4 \\
(1.30-) 1.35 \times 1.55\end{array}$ \\
\hline \multicolumn{6}{|l|}{$\begin{array}{l}\text { Terminal } \\
\text { elements of } \\
\text { pileipellis }\end{array}$} \\
\hline Length & $(36-) 45 \times 64(-78)$ & $(23-) 33 \times 59(-76)$ & $(40-) 47 \times 85(-88)$ & $(25-) 31 \times 84(-95)$ & $51 \times 76$ \\
\hline Width & $6.5 \times 9.5(-10.5)$ & $(5.5-) 6 \times 8$ & $(7-) 7.5 \times 11.5(-13.5)$ & $(9-) 10 \times 16(-18.5)$ & $12 \times 14.5$ \\
\hline \multicolumn{6}{|l|}{$\begin{array}{l}\text { Terminal } \\
\text { elements of } \\
\text { stipe hairs }\end{array}$} \\
\hline Length & $(11-) 15 \times 25(-64)$ & $(7-) 10.5 \times 61(-79)$ & $(25-) 31 \times 74(-79)$ & & \\
\hline Width & $2.2 \times 5.7$ & $2.5 \times 5(-5.5)$ & $(4.5-) 5 \times 7.5(-8)$ & & \\
\hline \multicolumn{6}{|l|}{$\begin{array}{l}\text { Elements of } \\
\text { stipititrama }\end{array}$} \\
\hline Length & $62 \times 119(-122)$ & $(20-) 43 \times 90(-103)$ & $(27-) 29 \times 110(-152)$ & $(125-) 135 \times 292(-367)$ & $(118-) 135 \times 446(-465)$ \\
\hline Width & $11 \times 23.5$ & $(7-) 8 \times 14.5$ & $(7.5-) 9 \times 13.5(-15)$ & $(14-) 17 \times 22(-31)$ & $(10-) 11.5 \times 19(-24)$ \\
\hline
\end{tabular}

Table 2. Comparison of microscopic characters of the studied Hygrocybe collections $(\mathrm{n}=10)$.

Specimens studied: CANADA. N.W.T. District of Keewatin, Rankin Inlet, Kudlulik Peninsula, 12.VIII.1971 Ohenoja \& M. Ohenoja (OULU F73587); 10.VIII.1971 Ohenoja \& M. Ohenoja (OULU*); 20.VIII.1971 Ohenoja $64 \&$ M. Ohenoja (OULU*). FINLAND Inari Lapland. Utsjoki, Gistuskaidi, 28.VIII.2012 Ronikier \& M. Ronikier (TB 12.008). NORWAY. Finnmark. Båtsfjord, Syltefjorddalen, at Kristoffervatnet, 5.VIII.1979 Høiland 219-79 \& Østmoe (O); Vadsø, Kiby, 6.VIII.1964 Heikkilä (OULU); Nesseby, Karlebotn, 25.VIII.1998 Ohenoja (OULU, two collections*); 29.VIII.2012 Ruotsalainen (TB 12.029). Nordland. Narvik, Gautelisfjell, 10.VIII.1974 Skifte 7/74 (TROM*). RUSSIA Tjumenskaja Oblast. Kara Sea, Cape Nyarusalye, 20.VIII.1996 Skifte (TB); 20.VII.1996 Peintner (TB); N. Urals, Labytnangi, Khaybej, 16.VIII.1996 Goldberg (TB); 16.VIII.1996 Knudsen (TB*).

Deviating collection: NORWAY. Finn- mark. Ekkerøy, St. Ekkerøy, E of the village, 20.VIII.1998 Ohenoja (OULU*).

Doubtful collection: NORWAY. Finnmark. Porsanger, Kistrand, Lakselv, Smørstad, way to Karasjok, 23.VIII.1965 Skifte, Lange \& B. Lange (TROM*).

Hygrocybe biminiata is well known from S. Norway (Finse area) (Kühner 1976, Borgen \& Arnolds 2004, Gulden 2005). However, it has not been recorded before from N. Norway, Finland, Arctic Canada nor from Arctic Russia, although its circumpolar occurrence could be expected. Judging from the data in Laursen et al. (1987: 276, description and fig. 5, as H. coccineocrenata) $H$. biminiata might well occur in Arctic Alaska.

The description is based on three well documented collections, which are very well in accordance with Kühner's diagnose and holotype collection 


$\begin{array}{llll} & \text { H. substrangulata } & \text { H. substrangulata var. } & \text { H. substrangulata } \\ \text { H. biminiata } & \text { var. substrangulata } & \text { substrangulata forma } & \text { var. rhodophylla }\end{array}$

\section{Spores}

Length

$(7.8-) 8.3 \times 10.8(-11.3)$

$(7.9-) 8.1 \times 11.8(-12.3)$

(9.6-)10.3 × 13.2

Width

(4.4-4.)4.9 × 6.6

$5.1 \times 7.4(-7.8)$

$(5.1-) 5.6 \times 6.9(-7.1)$

(7.8-)8.1 ×- 12.3(-12.5)

Average quotient $Q_{\text {m }}$

(1.45-)1.5 × 1.95

$1.25 \times 1.8(-1.95)$

(1.5-)1.55 × 2.1(-2.3)

(4.2-)4.7 × 7.8(-8.3)

$(1.45-) 1.5 \times 1.95(-2.3)$

Basidia

Length

(35-)44 × 49(-53)

$(44-) 46 \times 54(-58)$

$(38-) 47 \times 54(-65)$

(39-) $40 \times-53(-59)$

Width

$8 \times 9.5(-10)$

$5.1 \times 7.1$

$8.5 \times 10.5$

$(7-) 7.5 \times 10$

Terminal elements

of pileipellis

Length

$(26-) 27 \times 47(-61)$

(33-)36 × 65(-85)

(28-)40 × 96(-125)

(33-)36 × 88(-100)

Width

$6.5 \times 12.5(-13)$

$7.5 \times 15.5(-17.5)$

(9.5-)10.5 × 13.5(-15.5)

$(7-) 7.5 \times 20(-23)$

Terminal elements

of stipe hairs

Length

$(12-) 14 \times 66(-69)$

(15-)33 × 68(-84)

(38-)39 × 116(-127)

$(22-) 25 \times 73(-74)$

Width

(2.5.) $4 \times 6.5$

(4.6-) $5.5 \times 9.5(-11.5)$

$(5.5-) 6.5 \times 10.5(-11)$

$(4-) 6 \times 8.8(-9.6)$

Elements of

stipititrama

Length

(39-)40 × 93(-103)

(29-)51 × 145(-147)

(19-) $58 \times 125(-165)$

(29-)51 × 145(-147)

Width

$11 \times 15(-20)$

(9-) $10 \times 13(-13.5)$

(7.5-)8.5 × 13.5

$(7.5-) 8.5 \times 14.5(-17)$

Elements of

hymenophoral

trama

Length

$(22-) 38 \times 93(-95)$

$(41-) 43 \times 73(-102)$

$30 \times 64$

$(27-) 34 \times 100(-124)$

Width

$(5-) 7 \times 10.5(-12)$

$6 \times 11(-18.5)$

$5 \times 10.5$

$4.5 \times 11.5(-15)$

Table 3. Comparison of microscopic characters of the studied Hygrocybe collections $(\mu \mathrm{m}, \mathrm{n}=10)$.

from Norway (Finse), as well as with rich material from Greenland (cf. Borgen \& Arnolds 2004). Collection TB 12.008 was sequenced by M. Sasa et al. See also the relations between $H$. biminiata and $H$. substrangulata s.l. under the discussion further below.The microscopic features of the sparsely annoted Porsanger collection are in accordance with $H$. biminiata. However the habitat was given as "in wet mosses". Unfortunately, the material is very sparse, and not in good condition, hence we are unable to make a justified decision about its identity.
Hygrocybe substrangulata (P.D. Orton) P.D. Orton \& Watling var. substrangulata

- Figs. 5g, 1, p, 6c, e (right), Tables 1, 3

Pileus 8-15 mm wide, (plano-)convex with depressed centre, margin incurved, crenulate or not, occasionally slightly translucently striate towards margin, orange-yellow, orange, reddish orange, (near 6A6 - possibly slightly faded specimen), orange-red to red (7AB8, 8AB8, 9A8 / 9B8), minutely to distinctly fibrillose-squamulose, almost concolorous to more or less darker. 
Obviously immediately slightly darker, very soon fading in $10 \% \mathrm{KOH}$. Lamellae subdecurrent or emarginated with decurrent tooth, (sub) distant $(\mathrm{L}=12-16, \mathrm{l}=0-3)$, fairly thin to thick, initially almost whitish then pale yellowish, eventually with a reddish tinge (4A4, 4A6, 5A6). Stipe 11-25 $\times 2-2.5 \mathrm{~mm}$, subcylindric subflexuose, or with subinflated base, dry and dull, in apex concolorous with cap, about 9A7, towards base (yellowish-)pale, 4A8, 3A8, or unicolorous (more diluted than 4A4), slightly rugulose seen with a stereoscopic microscope. Context watery yellowish in central part, elsewhere subconcolorous, not very brittle, inodorous, taste none. Spores on average $9.2-9.8 \times 5.5-6.6 \mu \mathrm{m}$, ellipsoid, ovoid to oblong, in side view $0-50 \%$, in frontal view $0-20 \%$ constricted, $Q_{m}=1.49-1.75$. Gill edge fertile, cystidia not seen, basidia 4-spored (10/1): $\mathrm{Q}=(5.6-) 5.8-6.9$, predominantly (sub)clavate. Hymenophoral trama subregular, consisting of short, predominantly predominantly subcylindrical, rarely inflated elements. Pileipellis a trichoderm, terminal elements on average 45.8-58.2 $\times 8.9-12.2 \mu \mathrm{m}$, varying from subcylindrical to obliquely fusiform), not or hardly constricted at septa or in the midportion; pigment pale greyish ochre, narrow elements not observed. Stipitipellis a dry, subregular cutis with fairly few to many, very entangled hairs occurring in groups, frequently difficult to separate from each other, on average: $29.8-54.5 \times 6.4-7.0 \mu \mathrm{m}$, very variable in shape, some almost coralloid, subcylindrical to subinflated. Stipititrama subradial, consisting of short, (sub)cylindrical elements, on average $84.4 \times 11.6 \mu \mathrm{m}$. Clamp connections present. Oleiferous hyphae (occasionally) present.

Habitat: In sloping area with small rivulets and intermixed heath vegetation, in mainly wet mosses; two of them in Sphagnum; with e.g. Carex sp., Empetrum, Salix myrsinites, frequently near Betula nana.

Specimens studied: FINLAND. Koillismaa. Kuusamo, Alimmainen Hiidenlampi, 28.VIII. 2011 Campo (TUR-A). Inari Lapland. Utsjoki, upper S. slope of Nuvvus Áilegas, oroarctic zone, alt. 350-400 m, 25.VIII.2012 Ohenoja (TB 12.001); 30.VIII.2012 Ohenoja (TB 12.043); Borgen (TB 12.041); Ronikier \& M. Ronikier (KRAM F-49441 / TB 12.045); Kevo area, 21.VIII.1965 Skifte \& Siltanen (TROM); Utsjoki, church village, N. slope of Áilegas, 31.VIII.1973 Ulvinen (OULU). NORWAY. Finnmark. Karasjok, Baddasnjargga, 26.VIII.1968 Skifte 230/68 (TROM*), N of Vuollevuoppevarri, 3.VIII.1976 Hansen \& Wischmann (TRH); Nesseby, 7.IX.1970 Ulvinen (OULU), $1 \mathrm{~km}$ from Nesseby, 12.VIII.1967 Skifte 400/67 (TROM), Karlebotn, 7.VIII.1967 Skifte 276/67 (TROM*), Varangerbotn, 19.VIII.1965 Kankainen ( TUR), Varangerbotn, along the way to Skipagurra, 19.VIII.1975 Ulvinen (OULU), $1 \mathrm{~km} \mathrm{~W}$ of Varangerbotn, 7.IX.1970 M. Ohenoja (OULU, TUR); Tana, 18.VIII.1995 Ohenoja (OULU); Nordreisa, Leirbuktfjell, alt. 225 m, 16.VIII.1963 Skifte (TROM*). Troms. Harstad, up against Musevatn, 2.IX.1976 Vorren (TROM); Lyngen. Oksefjellet, at Danielelva, 6.IX.1965 Sivertsen (TROM); Lyngen, Karnesmyra below Leine, 29.VIII.1965 Sivertsen (TROM); 17.VII.1959 Sivertsen (TRH); Tromsø, Tromsdalen, N. side of the river, 23.VIII.1969 Skifte 111/69 (TROM*) RUSSIA. Tjumenskaja Oblast. Polar Urals, $10 \mathrm{~km}$ E of Labytnangi, 7.VIII.1992 Gøtzsche 92.081 (C*); Krasnij Charhanmei, $\sim 40 \mathrm{~km} \mathrm{NW}$ of Labytnangi, 16.VIII.1990 Knudsen 90.447 (C); Oktjabrskij, $\sim 10 \mathrm{~km} \mathrm{~N}$ of Labytnangi, 15.VIII.1990 Knudsen 90.373 (C); S. Jamal, along river Chadeta, $\sim 20 \mathrm{~km} \mathrm{E}$ of Biological Station, 12.VIII.1990 Knudsen 90.273 (C).

This variety is mainly boreal-arctic-alpine and quite frequent in Fennoscandia.The four described collections show some variation: pileus orange to orange-red, basidiocarps slender or not, lamellae decurrent or not and spores constricted or not. However, the spore-size, and the terminal elements of pileipellis are not very variable. H.substrangulata var. substrangulata was recorded from Finland (Korpilahti, Rimminjärvi, leg. E. Rald) by Borgen \& Arnolds (2004: 53), this has escaped the attention of Boertmann (2010) and Knudsen \& Vesterholt (2010).

Two collections (KRAM F-49441 and TB 12.042) with slender basidiocarps and arcuatedecurrent, initially almost white gills were reminiscent of pale-scaled collections of $H$. coccineocrenata or $H$. cantharellus, but had to be placed in H. substrangulata s.l. These deviations fall nicely into the variation described and figured in Borgen \& Arnolds (2004, figs. 3, 21, table 5). A closer study revealed that the former has microscopic features similar to typical collections 
of $H$. substrangulata. The latter (TB 12.042) has much larger spores (average $11.7 \times 6.4 \mu \mathrm{m}$, versus $9.5 \times 6.2 \mu \mathrm{m}$ ), slightly larger terminal elements of pileipellis and orange rather than reddish cap, and possibly closer gills; therefore we decided to describe it separately below in order to elucidate better the variation of the material referred to $H$. substrangulata s.l.

Hygrocybe substrangulata (P.D. Orton) P.D. Orton \& Watling, var. substrangulata forma

- Figs. 5h, m, 6d, e (left), Tables 1, 3

Pileus up to $14 \mathrm{~mm}$ wide, convex, orange about $7.5 \mathrm{~A} 7$, turning paler with $10 \% \mathrm{KOH}$, distinctly squamulose, these are darker towards centre, giving a general slightly darker impression. Lamellae (arcuate-) decurrent, or at most subdecurrent, fairly close $(\mathrm{L}=25, \mathrm{l}=1-3)$, rather broad, yellowish white near the edge, inwards $\sim 5 \mathrm{~A} 3$ transitional to $3 \mathrm{~A} 4$, in age slightly reddish. Stipe up to $32 \times 3.5 \mathrm{~mm}$, subcylindric or subinflated at base, dry, dull to slightly lustrous, distinctly rugulose under a stereoscopic microscope, in apex $\sim 8 \mathrm{~A} 7-6$, or to $\sim 7 \mathrm{~A} 5$ when young. Context subconcolorous, inodorous, taste mild. Spores $(20 / 2)$ on average $11.4-11.7 \times 6.1-6.4$ $\mu \mathrm{m}, \mathrm{Q}_{\mathrm{m}}=1.88$, predominantly oblong, occasionally ellipsoid, in side view $20 \%$, in frontal view < $10-15 \%$ constricted. Basidia 4-spored (10/1), Q $=(4.3-) 4.7-6.1(-6.9)$, (sub)clavate; edge fertile, cystidia not seen. Hymenophoral trama subregular, consisting of short, predominantly subcylindric elements. Pileipellis a trichoderm, elements $(25 / 2)$ on average $66.7-68.7 \times 11.7-12.3 \mu \mathrm{m}$, subcylindrical, subfusiform to subattenuated, with intracellular, very pale grey-brown pigment. Stipe hairs in groups, elements irregular and tortuous, on average 56.2-79.7 $\times 6.9-7.9$ $\mu \mathrm{m}$, proliferations also observed; stipititrama subregular, elements predominantly subcylindrical, on average $86.0 \times 10.3 \mu \mathrm{m}(12 / 1)$. Clamp connections present.

Habitat: At Nuvvus four specimens were found growing at rivulet on wet soil in mosses with Vaccinium uliginosum, V. vitis-idaea, Eriophorum scheuchzeri and Carex bigelowii. The Norwegian specimens grew in Sphagnum.
Specimens studied: FINLAND. Inari Lapland. Utsjoki, upper S. slope of Nuvvus Áilegas, oroarctic zone, 30.VIII.2012 Borgen (TB 12.042). NORWAY. Troms. Nordreisa, Sørkjosen, alt. 295 m, 11.VIII.1963 Skifte 2651 (TROM F-31749).

For characterization of this deviating material see (p. 12), observations under the previous description (p. 18). The annotated Norwegian collection deviates by having at most subdecurrent gills.

\section{Hygrocybe substrangulata var. rhodophylla} (Kühner) Boertm.

- Figs. 5i, n, 6f, Tables 1, 3

Pileus 9-20 mm, (plano-)convex, slightly depressed at centre, initially incurved, in younger specimen near 8A7, varying to "blackish red Lange (1923) N5" (Skifte, unpubl.), then darker, red, near 9A7 / 9B7 to 9B8, frequently slightly darker at centre and partly with darker scales; marginal zone eventually somewhat striate. Pileus surface possibly slightly darker with $10 \%$ $\mathrm{KOH}$, but very quickly slightly fading). Lamellae subdecurrent, subdistant $(\mathrm{L}=15-16, \mathrm{l}=0-2)$, fairly thin, pale yellow soon more or less reddish, e.g. $5 \mathrm{~A} 5$, in places $8 \mathrm{~A} 6$, to somewhat orangered. Stipe $18-25 \times 2-3 \mathrm{~mm}$, more or less roughened as seen with a stereoscopic microscope, typically reddish above with paler, yellowish base. Context inodorous, taste indistinct. Spores on average 9.9-11.5 × 5.1-6.7 $\mu \mathrm{m}, \mathrm{Q}_{\mathrm{m}}=1.65-$ 1.92 , ellipsoid to oblong, occasionally obovoid, in side view not or rarely constricted $(0-10 \%)$, in frontal view 5-15\% constricted. Edge fertile, cystidia not seen. Basidia 4-spored, (10/1): Q $=(4.1-) 4.3-6.5(-6.8)$, subcylindric to subclavate. Hymenophoral trama subregular, consisting of short, subcylindric elements. Pileipellis a trichoderm, terminal elements, on average 51.8-73.8 $\times$ 8.8-16.9 $\mu \mathrm{m}$, mainly subcylindric to subinflated, pigment intracellular, pale grey-brown, no narrow elements observed. Stipe hairs on average 47-54.5 × 6.3-7.0 $\mu \mathrm{m}$. Stipititrama subregular, made up of short elements, on average 77.9-84.4 $\times 11.6 \mu \mathrm{m}$. Clamp connections present.

Specimens studied: CANADA. N.W.T. District of Keewatin, Baker Lake, low arctic tundra, 16.VIII.1974 Ohenoja $97 \&$ M. Ohenoja 
(OULU*). FINLAND. Inari Lapland. Utsjoki, upper S. slope of Nuvvus Áilegas, oroarctic zone 30.VIII.2012 Ohenoja (TB 12.044). NORWAY. Finnmark. Karasjok, at the river, 18.VII.1961 Skifte 1934 \& Eckblad (TROM F-31595); 18.VII.1961 Skifte 1936 \& Eckblad (TROM*). Nordland. Hadsel, Teigen, N side of Jordvatn, E. side of the river, 10.IX.1985 Skifte 242/85 (TROM F-8761). Sør-Trøndelag. Hemne, Skrubbfjelet, alt. $400 \mathrm{~m}, 21$. VIII.2003 Oldervik $389.03\left(\mathrm{O}^{*}\right)$.

Doubtful records: NORWAY. Finnmark. Tana, Geidnogaissa, alpine zone, 21.VIII.1963 Suominen (TUR). Nordland. Rana, Virvatnet West, 8.IX.2005 Gaarder 4599 (O); Saltdal. Skjevlfjellet, 22.VIII.2001 Gaarder 2823 (O).

Habitat: Six gregarious, perfect specimens in moist site just above rivulet, on bare soil near Andromeda, Betula nana, Salix myrsinites, Eleocharis acicularis(?) and Carex sp. Three Norwegian records were growing in mosses with e.g. Dryas and Empetrum at rivers.

Hygrocybe substrangulata var. rhodophylla was known only from the Alps (Kühner 1976) and Greenland (Boertmann 1995; Borgen \& Arnolds 2004). In this paper we record it from Arctic Canada, an oroarctic site in Finland and boreal, subalpine and subarctic sites in Norway. There are elements in the description of the lamellae which indicate that a collection from Arctic Alaska (Laursen \& Ammirati \& Farr 3116, sub nom. $H$. aff. coccineocrenata, cf. Laursen et al. 1987: 280-281) might belong here. However, the description of the microscopic features is inconclusive.

The concept of this variety is in accordance with Kühner (1976) and Boertmann (1995), but only in part with Borgen \& Arnolds (2004, see below). Concerning the relations between $H$. substrangulata var. rhodophylla and var. substrangulata, see p. 18. The collections, on which the diagnosis is based, were filed correctly or as H. miniata. Although some of the field notes are deficient concerning the colour of the lamellae, we think it is justified to include them, as the documentation, including colours of well dried material, is very similar to fully documented, safely identified Greenland material.

In the study by Borgen \& Arnolds (2004) the presence and average width of stipe "hairs" were used as an important and easily observable tool to distinguish dried material of $H$. substrangulata (including var. rhodophylla) from $H$. biminiata (Tables 1, 3). The stipe covering showed some variation under a stereoscopic microscope, but no consistent difference between $H$. biminiata and H. substrangulatas.l. (including var. substrangulata forma and var. rhodophylla) was observed. The differences are thus only microscopical.

The single collection of Hygrocybe calciphila also turned out to have "stipe hairs". This was unexpected, because they were not found in a handful of collections from The Netherlands, England and Denmark (Borgen, unpublished). However, Arnolds (1986: 57) mentioned the scattered presence of "erect hyphal tips" (here interpreted as hairs rather than proliferations).

According to Borgen \& Arnolds (2004, fig. 3 ) the types of Hygrocybe substrangulata and $H$. coccineocrenata f. ambigua Kühner have large terminal elements in pileipellis, while $\mathrm{Hy}$ grophorus miniatus var. sphagnophilus Peck has somewhat smaller elements. As there are no collections sharing the typical ecology with the first one (moist sand dunes) its variation is not discussed here, but see Boertmann $(1995,2010)$ and Borgen \& Arnolds (2004). In the present material there are collections that match the types of the two others, in size and shape. The type of Hygrocybe rhodophylla Kühner has also large terminal elements (cf. Borgen \& Arnolds 2004). However, there was a large variation in the Greenland material and the same holds true for the material studied here.

Using a $\mathrm{KOH}$ spot test on the pileus, the pilei turned in general slightly darker but faded immediately afterwards. There was only little variation between the collections of $H$. biminiata, $H$. substrangulata var. substrangulata (including forma) and var. rhodophylla. A later test on fresh Danish material of $H$. miniata gave the same result, while the pileus did not fade in a fresh collection of $H$. turunda. The latter finding might indicate a difference to the other species, but should be tested on a larger material.

\section{On taxon variability and delimitations}

In the present subsection some species are more easily identified than others. The collections of 
Hygrocybe miniata, $H$. cantharellus, $H$. turunda and $H$. rubrolamellata could be identified fairly easily based on Borgen \& Arnolds (2004). The collection TROM F-31605 has marginally larger spores than described in the diagnosis of $\mathrm{H}$. calciphila (cf. Arnolds 1986: 57), on average $9.1 \times$ $6.0 \mu \mathrm{m}, \mathrm{Q}_{\mathrm{m}}=1.50$, versus 6.6-8.6 $\times 5.0-6.1 \mu \mathrm{m}$, $\left.\mathrm{Q}_{\mathrm{m}}=1.25-1.45\right)$. Borgen \& Senn-Irlet (1995: 99) give similar values in (low-)alpine Swiss material.

\section{The complex around $H$. biminiata and $H$. substrangulata, incl. var. rhodophylla}

In spite of the work by Borgen \& Arnolds (2004), in which a large number of collections within the $H$. substrangulata-complex were closely studied, identification has remained difficult in many cases due to unclear differences and different taxonomic concepts (cf. Boertmann 2010: 120, concerning the relations between $H$. biminiata and $H$. substrangulata). Based on later field studies in Greenland (2008) and a subsequent discussion with Boertmann (in litt.) Borgen became convinced that the concept of Hygrocybe substrangulata var. substrangulata was too narrow in that work, while the concept of var. rhodophylla was too broad. Against this background Borgen is now convinced that the macroscopic variation of var. rhodophylla, expressed in table 5, must be restricted to columns $2-4$ and rows $2-3$, while some of the remaining seem to be quite similar to what we consider here as "gracile, long-stiped" variants of $H$. substrangulata (probably including the "forma" - not yet checked). Although the types of H. substrangulata and Hygrophorus miniatus var. sphagnophilus are placed there as well, the description of the lamellae (cf. Orton 1960: 269-270 and Peck 1899: 857), is quite different. In our opinion there are three different problems, treated below, to be dealt with.

\section{The relations between $\boldsymbol{H}$. biminiata and H. substrangulata var. substrangulata}

Borgen \& Arnolds (2004) were convinced that $H$. biminiata and $H$. substrangulata were separate species due to narrower hairs and generally smaller terminal elements of pileipellis in the former than in the latter (Figs. 5k \& 51, Tables
1, 3). Hygrocybe biminiata prefers drier habitats than the varieties of $H$. substrangulata, which have been found growing on wet mossy soil (with or without Sphagnum). Due to these more or less sharp-cut differences, discussed below, we think that $H$. biminiata deserves specific recognition, but this idea should be confirmed with molecular analyses.

Borgen \& Arnolds (2004) were also convinced about macroscopic differences, although difficult to put in words and not included in the identification key. During the stay at Kevo the clear, vivid, to golden yellow gills, were striking in H. biminiata, and in accordance with the diagnose (Kühner 1976). The same holds true for the herbarium material, described as yellow, golden yellow to orange whenever annotated. The observed colour combinations in $H$. biminiata and $H$. substrangulata s.l., and the differences in habitus are given below, based on representative collections to elucidate more closely these observations (codes are explained in Table 1).

In Hygrocybe biminiata the following seven series (combinations) are present: Rydl, Xodl, Xydl, Xydl, Oydl, OydL, and Oydl. In Hygrocybe substrangulata var. substrangulata the following nine series are present: RoDL, RoDl, RodL, RyDL, XyDL, XyDl, OoDL, OyDL, and Oydl.

Overlapping series: Oydl (H. biminiata collections Ohenoja $64 \&$ M. Ohenoja and TB 96.015; $H$. substrangulata collection TB 12.043). However, these collections are neither intermediate concerning average size of the terminal elements of pileipellis, stipe hairs, cf. Table 1, nor ecology.

Finally, two collections are transitional concerning average size of the terminal elements of pileipellis. The annotated and photographed $\mathrm{Ca}-$ nadian collection (Ohenoja $64 \&$ M. Ohenoja) appears to be typical in all other respects. The deviating collection (Ekkerøy, 20.VIII.1998 Ohe$n o j a$ ) is in accordance with $H$. biminiata concerning its narrow stipe hairs (on average $4.4 \mu \mathrm{m}$ wide), and the ecology ("turfy tundra heath"), but it deviates by having gracile, long-stiped basidiocarps, decurrent lamellae and very narrow spores (on average $10.9 \times 5.5 \mu \mathrm{m}$ ) and we are not sure that it belongs here. Borgen \& Arnolds (2004: 49) found only very few transitions, as well, in spite of studying a large material. For variation on average spore size, terminal elements of pileipellis and stipe hairs, see table 1 . 


\section{The variation of Hygrocybe substrangulata s.l.}

The macroscopic features (habitus, colours and gill attachment) are very variable within the studied material. The same holds true for the average spore size, the percentage of spore constriction as well as average size of the terminal elements of pileipellis (Table 1). A closer survey on the variation of the present material is given below:

The four collections used in the description of Hygrocybe substrangulata var. substrangulata are encompassed by the series RyDL, XyDl, XyDL, and Oydl, while the series OoDL and OyDL are only present in the deviating form. In $H$. substrangulata var. rhodophylla four series are present, viz. RrDl, Rrdl, Rxdl, and RodL. The serie RodL encompasses two collections, TROM F-31596 and Ohenoja $97 \&$ M. Ohenoja, the former being identified as var. substrangulata, the latter as var. rhodophylla. The cap of a collection from Russia (Gøtzsche 92.081, series RyDL) is in accordance with var. rhodophylla, but not the gills. Could it be a transitional collection?

Borgen \& Arnolds (2004) demonstrated a large variation in largely the same features in $H$. substrangulata s.l., including the types of $H$. substrangulata, Hygrophorus miniatus var. sphagnophilus, H. coccineocrenata f. ambigua, and $H$. rhodophylla. The series of the second and the third of these types (RydL and Xydl) deviate from the nine series above, while the serie of the first $(\mathrm{RyDl})$ is represented also by Knudsen 90.273; while the last one ( $\mathrm{Rrdl})$ is also present in TB 12.044 .

For the time being we separate two collections (series (OoDL and OyDL) as H. substrangulata var. substrangulata forma, for morphologic reasons, as expressed by their series, and because their spores seem to differ from the "main" form, (see Table 1). Considering also the terminal elements of pileipellis they do not seem to be very similar to the types, cf. above (for details compare Table 1 with Borgen \& Arnolds (2004 figures 3, 21, table 5). This idea should of course be tested on a larger material, including e.g. the many representative collections included in Borgen \& Arnolds (2004), and by molecular methods.

\section{Observations on distributions}

The records of $H$. coccineocrenata from Inari Lapland (Ohenoja 1996) are based on wrong identifications. Moreover, many earlier records under this name indicate that large confusion with variants of $H$. substrangulata. $H$. coccineocrenata is not known from Greenland (Borgen $\&$ Knudsen 2004), in spite of being especially searched for. These findings indicate that the genuine $H$. coccineocrenata is rare, or possibly absent in the (sub)arctic regions. The small number of the records of $H$. turunda in N. Scandinavia seems astonishing, compared to its relatively frequency in S. Greenland, but the climate in Inari Lapland might be too continental for it. $\mathrm{H}$. Holien (pers. comm.) sent a convincing photo and indicated that there are several records of it from the lowland valleys at Tromsø; this might well indicate that it is more frequent in boreal sites. The epithet Hygrocybe miniata was been used for several species, including $H$. rubrolamellata and $H$. calciphila. It is quite likely that studies of additional old records would change their frequencies.

\section{Problematic identifications and use of names}

When the manuscript of Borgen and Arnolds (2004) was prepared, some information was retained for an additional paper on studies of types and other important material (cf. p. 56); however, this paper was never published. A little of the planned content is relevant for the present paper and is outlined below.

Orton's (1960) concept of Hygrocybe turunda was based on two collections. Watling kindly studied for Borgen \& Arnolds (2004) the collection (473), on which Orton's description and figures were based (Scotland, Inverness-shire, River Luineag, Rothiemurchus, 28.VIII.1955 Orton 473). Spores in this collections are on average: $10.2 \times 5.7 \mu \mathrm{m}, \mathrm{Q}_{\mathrm{m}}=1.75$, pileipellis, terminal elements, on average $46.8 \times 10.8 \mu \mathrm{m}$ and stipititrama elements on average $85.7 \times 15.4 \mu \mathrm{m}$. The short elements in the stipititrama do not match with the turunda-group. The spores, half of them being constricted according to the figure, do not either. H. turunda has only rarely constricted spores (cf. Borgen \& Arnolds 2004); whereas they are fre- 
quently constricted in $H$. substrangulata s.l. Surprisingly this indicates that an essential part of Orton's documentation must relate to a different species! In Greenland T. Borgen made a collection, identified as a variant of $H$. substrangulata, with largely the same features (Knudsen \& Borgen \& Petersen 93, cf. Borgen \& Arnolds 2004: 52); however, we do not know anything about the existence of stipe hairs in Orton's collection.

Kühner's type Hygrocybe coccineocrenata f. ambigua was studied by Arnolds \& Borgen after the printing of Flora Agaricina Neerlandica 2 (Arnolds 1990). In that work (p. 97) H. coccineocrenata f. ambigua and $H$. coccineocrenata var. sphagnophila were synonymized, but erroneously thought to be identical with a variant of $H$. coccineocrenata with pale brownish scales, which was described. Later this was corrected by Arnolds (1995), who published a study of Peck's type, see also Borgen \& Arnolds (2004). When Borgen \& Arnolds studied Kühner's type they demonstrated the short stipititrama elements, but at that time they did not yet pay attention to the presence of stipe hairs. However, Moreau studied the type (pers. comm.) and found broad stipe hairs like Borgen \& Arnolds (2004) did later in many other collections of $H$. substrangulata. This wrong synonymizing has been continued by Candusso (2002: 557) and Ludwig (2012: 325, 337).

\begin{abstract}
Acknowledgements: The authors thank E. Arnolds, D. Boertmann, H.F. Gøtzsche, G. Gaarder, H. Knudsen and P.-A. Moreau for valuable comments, A. Ruotsalainen for great assistance in the field during ISAM 9, and A. \& M. Ronikier for nice collections and photos, M. Sasa for preparing specimens for sequencing, K. Bendiksen, $\mathrm{H}$. Holien, G. Mathiassen, and J. Vauras for valuable information about the herbarium material, and the herbarium authorities of C, KRAM, O, OULU, TRH, TROM and TUR. L. Holmström and L. Kalleinen are thanked for technical help and L. Holmström especially for efforts in preparing the tables and graphics. E. Emmett is thanked for improving the English.
\end{abstract}

\section{References}

Arnolds, E. 1986: Notes on Hygrophoraceae VI. Observations on some new taxa of Hygrocybe. -Persoonia 13: 57-68.

Arnolds, E. 1990: Tricholomataceae trib. Hygrocybeae.In: Bas, C., Kuyper, Th.W., Noordeloos, M.E. \& Vellinga, E.C. (eds). Flora Agaricina Neerlandica 2. 137 pp. A.A. Balkema. Rotterdam.
Arnolds, E. 1995: Hygrophoraceae (Agaricales) in New York State and adjacent areas 1. Introduction and Hygrocybe subsect. Squamulosae. - Mycotaxon 33: $1-27$.

Boertmann, D. 1995: The genus Hygrocybe. - Fungi of Northern Europe 1. 184 pp. Svampetryk. Copenhagen.

Boertmann, D. 2010: The genus Hygrocybe, $2^{\text {nd }}$ revised edition. - Fungi of Northern Europe 1. 200 pp. Svampetryk. Copenhagen.

Borgen, T. \& Senn-Irlet, B. 1995: Hygrocybe glacialis spec. nov. and notes on subsection Squamulosae based on collections from Switzerland. - Documents Mycologique 25 (98-100): 91-102.

Borgen, T. \& Arnolds, E. 2004: Taxonomy, ecology and distribution of Hygrocybe (Fr.) P. Kumm. and $\mathrm{Ca} \sim$ marophyllopsis Herink (Fungi, Basidiomycota, Hygrocybeae) in Greenland. - Meddelelser om Grønland, Bioscience 54: 1-67.

Candusso, M. 1997: Hygrophorus s.l. - Fungi Europaei 6. 784. Alassio.

Gulden, G. 2005: A preliminary guide to the macromycetes in the Finse area, Hardangervidda, Norway (unpublished, available from the author).

Karsten, P.A. 1879: Rysslands, Finlands och den Skandinaviska halføns hattsvampar 1. Skifsvampar. - Bidrag Kännedomen av Finlands Natur och Folk 32: 1-571.

Knudsen, H., \& Vesterholt, J., (eds) 2012: Funga Nordica. Agaricoid, boletoid, clavarioid, cyphelloid and gasteroid genera. - 1083 pp. Nordsvamp. Copenhagen.

Kornerup, A. \& Wanscher, J.H. 1978: Methuen Handbook of colour. $3^{\text {rd }}$ edition. - Eyre Methuen, London.

Kühner, R. 1976: Agaricales de la zone alpine. Genus Hygrocybe (Fries) Kummer. - Bulletin de Trimestr. Societé Mycologique France 92: 455-515.

Lange, J.E. 1923: Colour chart, in studies in the Agarics of Denmark. - Dansk Botanisk Arkiv 4: 1-12. (unpaginated)

Lange, M., \& Skifte, O. 1967: Notes on the macromycetes of northern Norway. - Acta Borealia A 23: 1-51. Universitetsforlaget Tromsø / Oslo.

Laursen, G.A, Ammirati, J.F. \& Farr, D.E. 1987: Hygrophoraceae from Arctic and Alpine tundra in Alaska. - In: Laursen, G.A, Ammirati, J.F. \& Redhead, S.A. (eds): Arctic and Alpine Mycology 2. The second International Symposium on Arcto-Alpine Mycology 1987: 273-286. Plenum Publishing Corporation. New York.

Ludwig E., 2012: Die übrigen Gattungen der Agaricales mit weissem Sporenpulver. - Pilzkompendium 3: 1-881. Fungicon-Verlag. Berlin.

Ohenoja, E. 1996: A check-list of larger fungi in Inari Lapland (NE Finland) and in Finnmark (NE Norway). - Kevo Notes 11: 1-44.

Orton, P.D. 1960: New checklist of British agarics and boleti, Part 3. Notes on genera and species in the list. - Transactions of the British Mycological Society 43: 159-439.

Peck, C. 1901: Report of the State Botanist. - N. Y. State Museum Annual Report 53: 823-864. 
